\title{
A Deep Learning Approach to Retrieving 3D Structure Information from High Resolution Time-Resolved TEM Images
}

Ramon Manzorro ${ }^{1}$, Matan Leibovich ${ }^{2}$, Joshua Vincent ${ }^{1}$, Sreyas Mohan ${ }^{2}$, David Matteson ${ }^{3}$, Carlos Fernandez-Granda ${ }^{2}$ and Peter Crozier ${ }^{4}$

${ }^{1}$ Arizona State University, United States, ${ }^{2}$ New York University, United States, ${ }^{3}$ Cornell University, United States, ${ }^{4}$ Arizona State University, Tempe, Arizona, United States

The analysis of nanoparticles has enjoyed a continuously increasing interest, with applications such as catalysis, medicine or optoelectronics. The physical and chemical properties of these particles rely on their exact 3D structure, and multiple approaches have been developed to extract this information. The most accurate and reliable procedure for retrieving the nanoparticle morphology is scanning transmission electron microscopy (S/TEM) tomography, which performs even at the atomic scale [1]. However, tomographic techniques require acquiring at least two different image projections from different zone axes, demanding stable and stationary systems. This also limits the temporal resolution, necessary to capture dynamics in the nanoparticle 3D structure, which can be critical to the underlying functionality of interest. Other approaches use the intensity of a single high-resolution STEM or TEM image, combined with very precise image simulations $[2,3]$. Here too, however, temporal resolution is limited by the signalto-noise ratios required in order to reliably measure the intensity of each atomic column.

Convolutional neural networks have the potential to extract the morphology of a nanoparticle from a single image by determining the number of atoms per column. Pioneering work on 3D shape reconstruction of gold nanoparticles using machine learning was first done on simulated TEM images [4] followed by experimental data [5]. Still, in the presence of low doses, i.e., poor signal-to-noise ratios, the reported results are not accurate. In order to address these issues, we propose a convolutional neural network-based algorithm to retrieve 3D atomic structure, namely the atomic column heights, even in the presence of strong noise. At present we have focused our initial efforts on cerium dioxide (ceria, $\mathrm{CeO} 2$ ) nanoparticles due to the importance in catalytic energy conversion processes.

The general sketch of the training and evaluation process is depicted in Figure 1. The neural network is based on the Unet architecture. Training is done using simulated data capturing the complexities one might encounter in dynamical nanoparticles, including variations in thickness, point defects or modifications of electron-optical parameters. In addition, Poisson noise is added, which models the shot noise encountered experimentally on fast direct electron detectors. The output of the network is matched to a mask based on the 3D structure data file of the nanoparticle. The network parameters are trained by minimizing the output error using a weighted loss function.

Our approach achieves promising preliminary results on simulated data with low signal-to-noise ratios. Figure 2a illustrates a representative input where the atomic columns of the simulated image are hardly visible. The output of the image, Figure $2 \mathrm{~b}$, detects all these columns and provides an estimate of their thickness. The estimates are highly accurate (compare to ground truth in Figure 2c), except for a small artifact indicated by a red arrow. In future work, the network will be applied to real TEM data, allowing us to resolve the 3D structure of nanoparticles which dynamically changes throughout the image acquisition process [7]. 


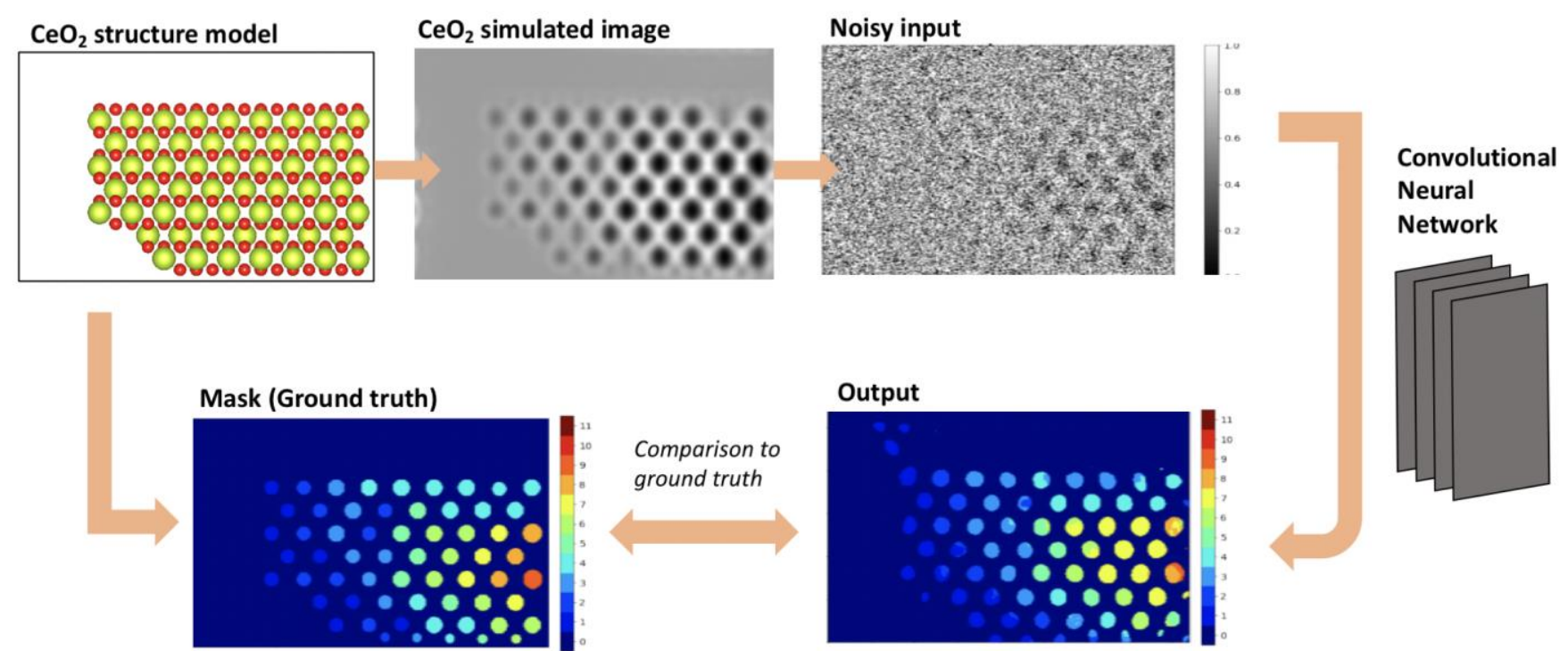

Figure 1. Sketch illustrating the general procedure to extract morphological information using through convolutional neural networks. The structural model (top, left) is used to generate the simulated image (top, middle) and the ground truth containing the 3D information (bottom, left). Then, the simulated image is modified with the addition of Poisson noise, which is input into the network, which produces an estimate of the column location and occupancy (bottom, right).

a) Noisy simulated image input

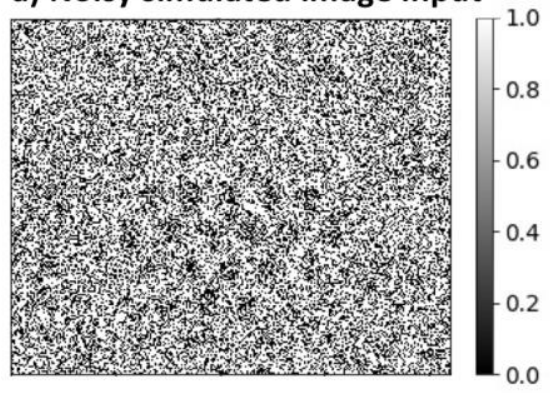

b) Output

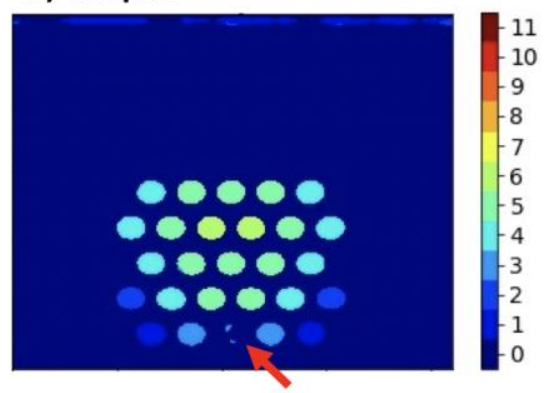

c) Mask (ground truth)

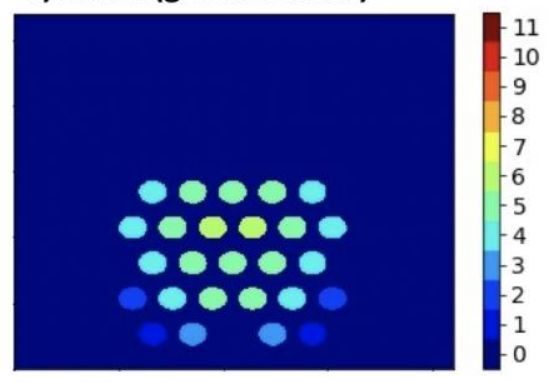

Figure 2. a) Representative noisy image simulation of a $\mathrm{CeO} 2$ nanoparticle, which is input to the proposed network. b) Output of the network, which provides an estimate of the number of atoms per column. c) Mask obtained from the structural model used as ground truth to evaluate the performance of the output.

References

[1] Van Aert, S., et al. Nature, 2011. 470, p. 374-377.

[2] Li, Z. Y., et al. Nature, 2008. 451, p. 46-48.

[3] Jia, C. L., et al. Nature Materials, 2014. 13, p.1044-1049.

[4] Madsen, J., et al. Adv. Theory Sim, 2018. 1, 1800037

[5] Ragone, M., et al. Comput. Mat. Sci., 2020. 180, 109722.

[6] Vincent, J. L., et al., pre-print at: https://arxiv.org/abs/2101.07770, 2021.

[7] We gratefully acknowledge support of NSF grant CBET-1604971, NRT-1922658, CCF-1934985, OAC-1940097, OAC-1940124 and OAC-1940276, and the facilities at ASU's John M. Cowley Center for High Resolution Electron Microscopy 